\title{
Comment 10.1
}

HENRI J.M. THEUN IS S EN

The current chapter studies how to successfully transfer knowledge from public research organizations to companies. Clearly, this depends, among other things, on the technological and related capabilities of firms and public research organizations, the gap between these capabilities, and the industrial structure of a country.

In this commentary I would like to provide some insight on the basis of my experience in the past in science, business and valorization at the University of Maastricht and the Brightlands Maastricht Health Campus.

Valorization is a term that is used in the Netherlands and some other countries to indicate the process through which knowledge from academic institutions is made available and relevant to society. In our practice, we attempt to translate this knowledge in the form of licenses, spinoffs and alliances with companies, or a combination of these. In fact, a license is always provided to the company, be it a spinoff of the university itself or a third party (i.e., a company not affiliated to the university). In this respect, licensing is crucial to the knowledge transfer process. It may be given either as a "standalone" asset in the form of a licensing agreement or in the context of a broader collaboration agreement.

The decision whether to grant a license to an already existing company or to a yet-to-be-established spinoff of the university is taken based on many factors, such as the mere availability of a licensee, bargaining power, match between the parties, preferences of stakeholders at the university and the valorization office, the nature and maturity of the technology, etc. The overriding argument is, however, the estimated overall probability of success that the technology will actually get to market in favor of customers or - in our case - patients. Hence, in principle, we would prefer to license our IP to firmly established companies that would develop the technology to maturity and bring it to 
market, while we receive a reasonable return on our investment in the research and the IP ensuing from it.

However, the chance of finding such a perfect licensee is slim, and, in practice, we have seen only a few such examples. This may be related to the subject of the science and the licensing opportunities that emerge from our knowledge institution. Indeed, we have in the past generated relatively few technology platforms, let alone ones that are able to generate products, such as monoclonal antibodies for therapy. That situation is gradually changing and hence may result in more straightforward licensing deals. Instead, the most frequent form of valorization of IP occurs via licensing to our own spinoff companies.

Having said this, we would very much like to increase our performance in terms of licensing to companies. There are many activities one might undertake to enhance the probability that companies would license our IP, such as putting even more effort into showcasing our opportunities, e.g., via websites, portals, and other marketing tools.

One aspect that makes straightforward licensing difficult is tacit knowledge. It often occurs that there is a long scientific and maybe even business history behind an emerging licensing opportunity. The moment the company is asked to have a look at the opportunity there is a huge lack in knowledge, understanding, and experience with the matter at hand. This seriously hampers the closing of a deal based on just one piece of information (i.e., the IP or the patent). In my daily practice at the company I worked for, I never licensed any isolated piece of IP from an academic partner outside the realm of an established collaboration. Also, besides the tacit knowledge issue there is yet another very practical problem: if a company scientist tells management that the IP at hand is interesting and should be licensed, it implies a risk that external technology may be better than their own and that the latter may be abandoned!

Based on my previous experience in the pharma industry, I believe that establishing an alliance with an industrial partner is the preferred route toward effective IP licensing. This would then be a separate paragraph in the collaboration agreement in which the company has an option to license or even acquire IP emerging from the collaboration. This would enable the company to develop and sell products covered by that IP. This should give the company sufficient comfort to use the results from the collaboration for their benefit.

It goes without saying that such an arrangement would require a reasonable return for the knowledge institution. Depending on the nature and value of the IP and the preference of the partners, this could 
be in the form of (additional) sponsored research or upfront, milestone, or royalty payments. In practice, it turns out that royalties are sometimes a no-go for companies. This is the most cumbersome part of the negotiation, as it directly affects product margins. However, it is reasonable as well as realistic to address this issue during negotiations. Both parties should make an effort to discuss this matter in good faith. Also, they should keep in mind that IP is a means, not a goal, and therefore should be treated with proportionate priority, especially when it comes to early IP emerging from an academic collaboration.

It is important that the inventor of the IP and their department gets a fair share of the return made on IP revenues. In our institution we split any revenues from IP in three equal parts, i.e., a third each for the inventor(s), department(s) and the valorization organization itself. Such an incentive is important to motivate scientists to go the extra mile, often after daily work or at the weekend. At the same time, it is a great deal for the department as well, as revenues are spent on new research that, once again, could generate novel IP.

Even though I have described the experiences and practices that we have at our institution in the Netherlands, I believe that the main issues that I have mentioned are similar in many other developed countries. The operational implementation of these issues and policies will, however, be different from one country to another. In any case, the overall guiding principle is that IP is a major driving force for valorization, knowledge transfer, and innovation across the globe! 